\title{
Robustness of command input preshaping technique applied to residual vibration reduction
}

\author{
Paolo Pennacchi \\ Dipartimento di Meccanica, Politecnico di Milano, Via La Masa, 34, I-20156, Milano, Italy \\ Tel.: +3902 2399 8440; Fax: +3902 2399 8492; E-mail: paolo.pennacchi@polimi.it
}

\section{Ricordo di Bruno Piombo}

Ho vissuto insieme a Bruno Piombo uno dei momenti più dolorosi della storia recente. Il giorno 11 settembre 2001, insieme ad altri colleghi italiani, ci trovavamo a Pittsburgh per il 2001 ASME Design Engineering Technical Conference. L'enorme tensione del momento, il senso di vacuità che aveva pervaso tutti, l'orrore per la viltà dell'atto, la precarietà di essere stranieri e di non sapere quando poter tornare in patria trovavano conforto in Bruno Piombo, perché egli, al di là di essere valente ingegnere e scienziato, era uomo di saldo spirito. Mi sembra significativo e doveroso contribuire al suo ricordo in questa sede con una memoria su un tema affine a quello presentato, con i colleghi Mimmi, Frosini e Rottenbacher, in occasione di quella conferenza.

\begin{abstract}
Open-loop control techniques, such as command input preshaping, are quite sensible to modelling errors and noise. In the paper, an analysis and a discussion about the robustness of the technique with respect to the errors than can affect estimation of the values of the system parameters is presented.
\end{abstract}

\section{Introduction}

Although preshaping technique applied to residual vibration reduction has been proposed since some fifty years, being the O.J.M. Smith's first work published in 1957, its application in system and structure control is more recent and several papers have been published since 1989. The application of the technique is mainly in the field of robotics in regards to linkages characterized by light and flexible links and similar fields in which both the end-effector lack of vibration at the end of the motion and the accuracy in the positioning are important, such as in pick-and-place systems, co-ordinate measurement machines or gantry cranes.

Rather than list the papers appeared in literature, which would require several pages, it is better to cite the web page of the School of Mechanical Engineering, Georgia Institute of Technology [1] that contains the most important references.

The main idea of the method is that, by considering the response of a linear system to a pulse of a certain magnitude $A$, the pulse can be split into a sequence of smaller pulses of amplitudes $A_{j}$ delayed in time. By suitably calibrating the delays $\Delta t_{j}$ between the pulses, superposition of the responses leads to the vibration cancellation. 


\section{Robustness of command input preshaping technique}

Since preshaping technique is a feed-forward technique, it requires a rather precise identification of the system eigenvalues to be robust. In this paper the problem of the robustness is analysed by evaluating systematically the effects of the errors in the eigenvalues estimate.

In several papers, for instance in $[6,8]$, the authors observe that the increase of the pulse number improves the robustness of the system even though this introduces delays in the response [4]. However, in order to analyse also this aspect it is necessary to express the amplitude $A_{j}$ and the application time $t_{j}$ of the pulses for a general sequence composed by a general number $N$ of pulses. The general expressions of the time sequence $t_{j}$ and pulse amplitudes $A_{j}$ are presented in [5]:

$$
\begin{aligned}
t_{j} & =\frac{(j-1) \pi}{\omega \sqrt{1-\zeta^{2}}} \text { with } j=1, \ldots, N \\
A_{j} & =\frac{\left(\begin{array}{c}
N-1 \\
j-1
\end{array}\right) \kappa^{j-1}}{(1+\kappa)^{N-1}}=\frac{\left(\begin{array}{c}
N-1 \\
j-1
\end{array}\right) \eta^{N-j}}{(1+\eta)^{N-1}}, \kappa=e^{-\frac{\zeta \pi}{\sqrt{1-\zeta^{2}}}}, \eta=e^{\frac{\zeta \pi}{\sqrt{1-\zeta^{2}}}} \text { with } j=1, \ldots, N
\end{aligned}
$$

where $\omega$ is the natural frequency, $\zeta$ the damping ratio and the binomial coefficients are given by:

$$
\left(\begin{array}{l}
n \\
r
\end{array}\right)=\frac{n !}{r !(n-r) !}
$$

Should be also observed that the procedure, used in [5] to prove mathematically Eqs (1) and (2), requires the use of the partial derivatives of order $N-2$ with respect to the frequency of the zero residual vibration constraints, that are defined as:

$$
\begin{aligned}
& \sum_{j=1}^{N} A_{j} e^{-\zeta \omega\left(t_{N}-t_{j}\right)} \cos \left(t_{j} \omega \sqrt{1-\zeta^{2}}\right)=0 \\
& \sum_{j=1}^{N} A_{j} e^{-\zeta \omega\left(t_{N}-t_{j}\right)} \sin \left(t_{j} \omega \sqrt{1-\zeta^{2}}=0\right.
\end{aligned}
$$

These partial derivatives, when are equal to zero, have the following general expression:

$$
\begin{aligned}
& \sum_{j=1}^{N} A_{j} t_{j}^{q} e^{-\zeta \omega\left(t_{N}-t_{j}\right)} \sin \left(t_{j} \omega \sqrt{1-\zeta^{2}}\right)=0 \text { with } q=1, \ldots, N-2 \\
& \sum_{j=1}^{N} A_{j} t_{j}^{q} e^{-\zeta \omega\left(t_{N}-t_{j}\right)} \cos \left(t_{j} \omega \sqrt{1-\zeta^{2}}\right)=0 \text { with } q=1, \ldots, N-2
\end{aligned}
$$

The use of Eqs (6) and (7) implies immediately a reduction of the sensitivity to natural frequencies variation when the number of pulses increases. In [8] it is observed that also the partial derivatives with respect to the damping ratio have the same general expression of Eqs (6) and (7) when are equal to zero and it is stated that "if the input sequence is insensitive to error in natural frequency estimates, it is also insensitive to error in damping ratio estimates". This purely qualitative statement is confirmed in quantitative terms in the following and it is also shown that the actual damping ratio value is influent on the robustness.

It is also worth to remember that the preshaping technique can be applied by using modal superposition on structures having a linear behaviour such as flexible links as shown for instance in [9] by means of numerical simulations and in [2] in test rigs.

The preshaping method requires the identification of frequency and damping of each mode that has to be controlled, i.e. whose residual vibration at the end pulse sequence is null. By considering one mode at time, if its actual natural 
frequency $\omega_{1}$ and damping ratio $\zeta_{1}$ differ from the estimated frequency $\omega$ and damping ratio $\zeta$ that are used to define the time sequence $t_{j}$ and the pulse amplitudes $A_{j}$, obviously the response of the mode at the end of the $N$ pulse sequence, given by:

$$
y(t)=\sum_{j=1}^{N} B_{j} \sin \left(\alpha t+\phi_{j}\right)=A_{\mathrm{amp}} \sin (\alpha t+\psi)
$$

has its amplitude $A_{\mathrm{amp}}$ different from zero. The difference between the actual system eigenvalues and the estimated ones can be due to several reasons such as identification uncertainties, unpredictable changes in the physical system or a position change as in the case of flexible manipulators composed by several links.

The theory of this method assumes that the system response can be considered acceptable if the maximum overshoot value, or similarly the residual vibration amplitude, is conveniently limited. The definition of the maximum overshoot should usually depend on the application; however, well established and general criteria (see e.g. [3]) set an acceptable value of 2 or $5 \%$ of the overall displacement. The last value is chosen in this paper even if the actual numerical value can be set as much as one likes.

A numerical analysis of the effect of using $N$ pulses on a system can be done by considering that the time sequence $t_{j}$ and pulse amplitudes $A_{j}$, calculated using the estimated values $\omega$ and $\zeta$ by means of Eqs (1) and (2), do not change their value. On the contrary, the coefficients of the system response to each pulse (see Eq. (8)) take into consideration the actual values of frequency $\omega_{1}$ and damping ratio $\zeta_{1}$ and become:

$$
\hat{B}_{j}=\frac{A_{j}}{m \omega_{1} \sqrt{1-\zeta_{1}^{2}}} e^{-\zeta_{1} \omega_{1}\left(t-t_{j}\right)}, \quad \hat{\alpha}=\omega_{1} \sqrt{1-\zeta_{1}^{2}}, \quad \hat{\phi}_{j}=-\omega_{1} \sqrt{1-\zeta_{1}^{2}} t_{j}
$$

The sensitivity analysis of the overshoot can be directly done on $A_{\mathrm{amp}}$, since it is already normalized:

$$
\frac{A_{\mathrm{amp}}}{\sum_{j=1}^{N} A_{j}}=A_{\mathrm{amp}}=\sqrt{\left(\sum_{j=1}^{N} \hat{B}_{j} \cos \hat{\phi}_{j}\right)^{2}+\left(\sum_{j=1}^{N} \hat{B}_{j} \sin \hat{\phi}_{j}\right)^{2}}
$$

In fact, due to the condition of non amplification of the response, the sum of all the amplitudes of the pulses have to be equal to one:

$$
\sum_{j=1}^{N} A_{j}=1
$$

Moreover, to show the effect of the error between the actual and the estimated frequency and damping, it is convenient to consider their difference:

$$
\begin{aligned}
& \omega_{1}-\omega=\Delta \omega \\
& \zeta_{1}-\zeta=\Delta \zeta
\end{aligned} \rightarrow \begin{aligned}
& \omega_{1}=\omega+\Delta \omega \\
& \zeta_{1}=\zeta+\Delta \zeta
\end{aligned}
$$

Therefore, values over and below zero have to be interpreted as negative and positive delta around the actual value.

If a case with low damping ratio $(\zeta=0.1)$ and unitary (normalized) natural frequency is considered $(\Delta \omega$ are therefore percentage values), the diagrams given by Eq. (10) for $N=2$ to $N=5$ are reported in Fig. 1 for a maximum overshoot of $5 \%$. The surfaces are one inside the other, like "barks", being the inner corresponding to $N=2$ and the outer to $N=5$. The area sliced by the plane $A_{\text {amp }}=0.05$ indicates the set of $\Delta \omega$ and $\Delta \zeta$ values which limit the maximum overshoot to the required $5 \%$ value. More in general, the relation between the number $N$ of pulses, the desired maximum overshoot $\bar{A}_{\mathrm{amp}}$ and the error margins $\Delta \omega$ and $\Delta \zeta$ between the actual and the estimated frequency and damping (Fig. 2) can be obtained by solving:

$$
\sqrt{\left(\sum_{j=1}^{N} \hat{B}_{j} \cos \hat{\phi}_{j}\right)^{2}+\left(\sum_{j=1}^{N} \hat{B}_{j} \sin \hat{\phi}_{j}\right)^{2}}=\bar{A}_{\mathrm{amp}}
$$

which it is equal to slice surfaces of Eq. (10) with the plane $A_{\mathrm{amp}}=\bar{A}_{\mathrm{amp}}$.

Similarly, Fig. 3 shows the diagrams of Eq. (10) for $N=2$ to $N=5$ in a case with high damping ratio $(\zeta=0.5)$, unitary natural frequency and same maximum overshoot.

From the analysis of the diagrams in Figs 1 and 3, some considerations can be inferred. 


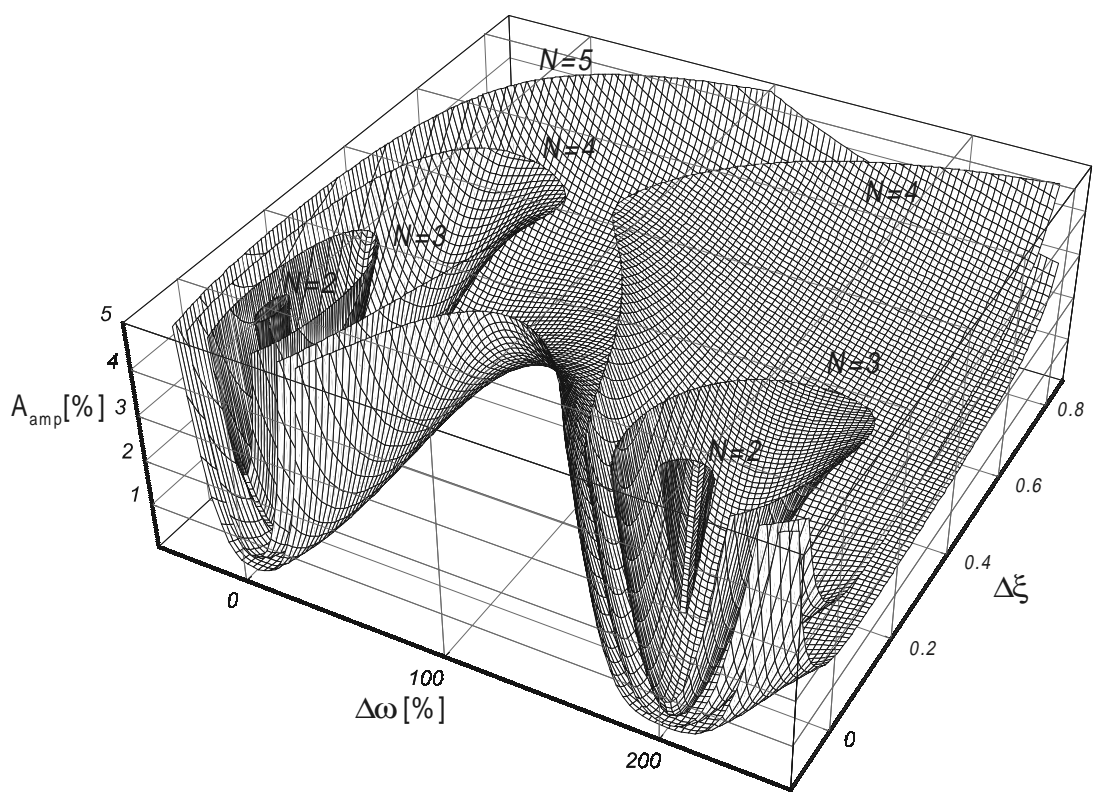

Fig. 1. Amplitude at the end of the pulse sequence with maximum overshoot $A_{\mathrm{amp}}<0.05$, low damping ratio $(\zeta=0.1)$.

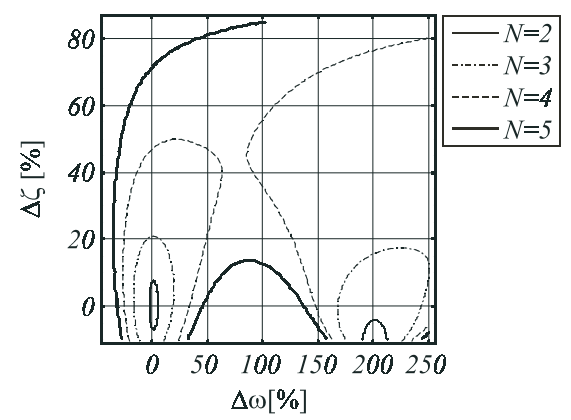

Fig. 2. Error margins between actual and estimated frequency and damping with maximum overshoot $A_{\mathrm{amp}}<0.05$, low damping ratio $(\zeta=0.1)$.

i) An increase of the number $N$ of pulses allows greater uncertainties in the identification of system parameters natural frequencies $\omega$ and damping ratio $\zeta$ - to be tolerable and even when $N=5$, an overestimation of the natural frequency appears to be unimportant: the superposition of the response of each pulse leads the total response to satisfy the limit on the overshoot. Similar results, are obtained with more restrictive overshoot limits, i.e. considering a slicing plane given by $A_{\mathrm{amp}}<0.05$. Moreover the value of the overshoot value could allow multiple and not connected (see $N=2$ to 4 in Fig. 1 and $N=2$ in Fig. 3) nor convex acceptable sets of $\Delta \omega$ and $\Delta \zeta$ to exist. The results are different in case of under estimation of the natural frequency since the results are more sensitive to the error.

ii) Given the number of the pulses, the case with high damping ratio permits greater errors than the case with low damping ratio.

iii) The sensitivity analysis has to be performed simultaneously on the frequency and on the damping. Previous studies in literature limit the consideration of a parameter at a time but the analysis presented here shows the cross influence of the two parameters. Moreover it could be convenient using a different number of pulses for different modes depending on their own damping ratio.

First and second considerations can be considered as a "rule of thumb", but they have also a mathematical proof, which shows that asymptotically, i.e. for $N \rightarrow \infty$, the method is robust for any error on both the frequency and the 


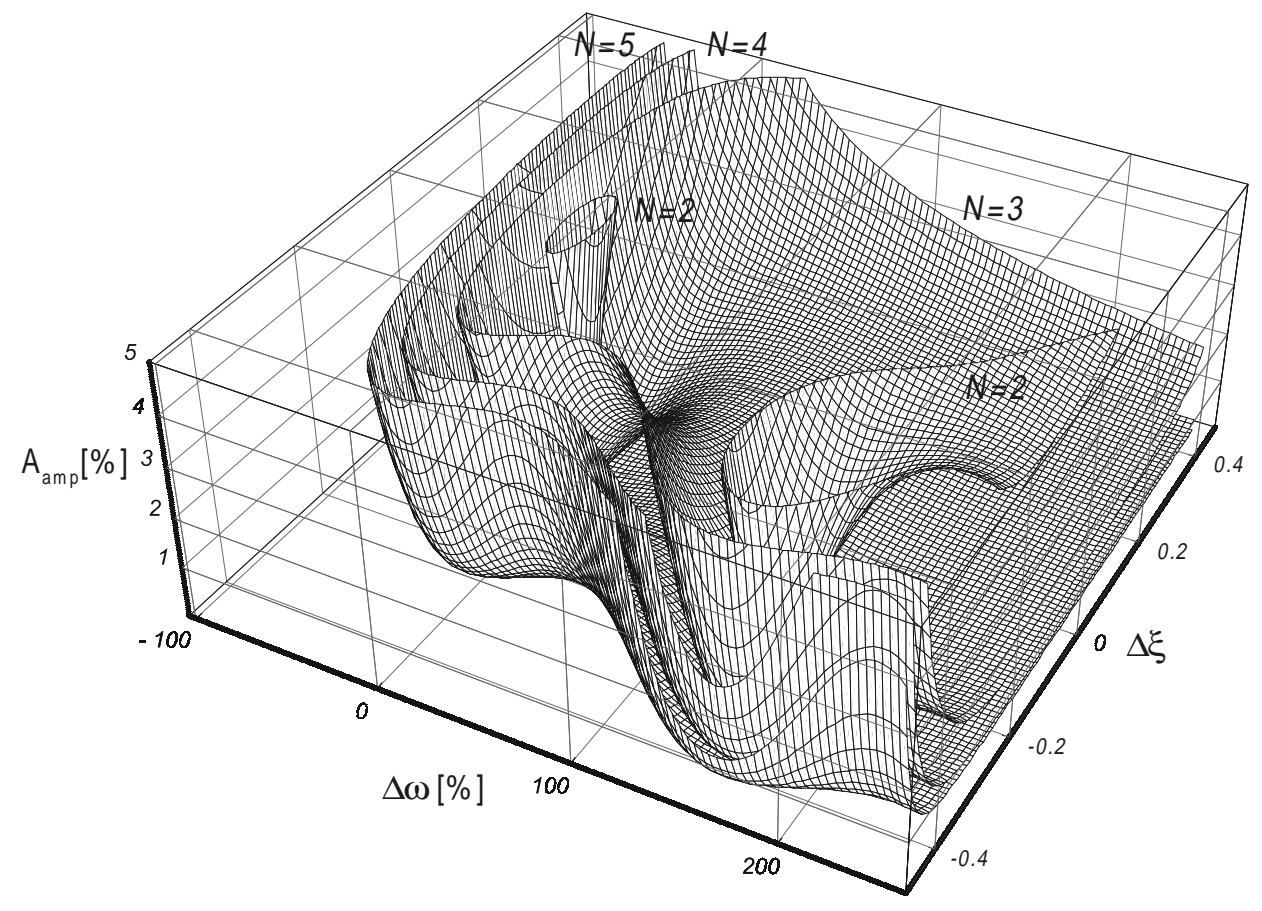

Fig. 3. Amplitude at the end of the pulse sequence with maximum overshoot $A_{\mathrm{amp}}<0.05$, high damping ratio $(\zeta=0.5)$.

damping ratio. This is equivalent to rewrite Eq. (10) as:

$$
A_{\mathrm{amp}}=\sqrt{\left(\sum_{j=1}^{\infty} \hat{B}_{j} \cos \hat{\phi}_{j}\right)^{2}+\left(\sum_{j=1}^{\infty} \hat{B}_{j} \sin \hat{\phi}_{j}\right)^{2}}=0 \forall \omega_{1}, \zeta_{1}
$$

To prove that the sum of the two series in Eq. (14) converges to zero, their $N^{\text {th }}$ partial sums have to be calculated. Since this task is very complicated, it has been performed by the support of MATHEMATICA ${ }^{\complement}$ software:

$$
\begin{aligned}
& A_{\mathrm{amp}}(N)=\sqrt{\left(\sum_{j=1}^{N} \hat{B}_{j} \cos \hat{\phi}_{j}\right)^{2}+\left(\sum_{j=1}^{N} \hat{B}_{j} \sin \hat{\phi}_{j}\right)^{2}} \\
& =\sqrt{-\frac{e^{\frac{\left.4 \pi \omega_{1} \zeta_{1}+i \sqrt{1-\zeta_{1}^{2}}\right)}{\omega \sqrt{1-\zeta^{2}}}}\left(e^{-\zeta_{1} \omega_{1}}\right) \frac{2 \pi}{\omega \sqrt{1-\zeta^{2}}}\left(1+e^{\frac{\pi \zeta}{\sqrt{1-\zeta^{2}}}}\right)^{2}}{\left(e^{\frac{\pi \zeta}{\sqrt{1-\zeta^{2}}}}+e^{\frac{\pi \omega_{1}\left(\zeta_{1}+i \sqrt{1-\zeta_{1}^{2}}\right.}{\omega \sqrt{\left(1-\zeta^{2}\right)}}}\right)\left(e^{\frac{\pi \zeta_{1} \omega_{1}}{\omega \sqrt{1-\zeta^{2}}}}+e^{\frac{\pi\left(\zeta \omega+i \omega_{1} \sqrt{1-\zeta_{1}^{2}}\right)}{\omega \sqrt{1-\zeta^{2}}}}\right) m^{2}\left(\zeta_{1}^{2}-1\right) \omega_{1}^{2}}} \\
& \sqrt{e^{\frac{-2 N \pi \omega \zeta_{1}}{\omega \sqrt{1-\zeta^{2}}}}\left(1+e^{-\frac{\pi \zeta}{\sqrt{1-\zeta^{2}}}}\right)^{-2 N}\left(1+e^{\frac{\pi\left(-\zeta \omega+\zeta_{1} \omega_{1}-i \omega_{1} \sqrt{1-\zeta_{1}^{2}}\right)}{\omega \sqrt{1-\zeta^{2}}}}\right)^{N}\left(1+e^{\frac{\pi\left(-\zeta \omega+\zeta_{1} \omega_{1}+i \omega_{1} \sqrt{1-\zeta_{1}^{2}}\right)}{\omega \sqrt{1-\zeta^{2}}}}\right)^{N}}
\end{aligned}
$$

Equation (15) can be split into two parts: the first squared root expression includes numerical terms that can be considered as constant, while the second squared root expression collects the terms that depend on $N$. To assure the asymptotic behaviour, i.e. the convergence of the series, the limit can be calculated on the last part only: 


$$
\begin{aligned}
\lim _{N \rightarrow \infty} A_{\mathrm{amp}}(N) & =\lim _{N \rightarrow \infty} \sqrt{e^{\frac{-2 N \pi \omega_{1} \zeta_{1}}{\omega \sqrt{1-\zeta^{2}}}}\left(1+e^{-\frac{\pi \zeta}{\sqrt{1-\zeta^{2}}}}\right)^{-2 N}} \\
& \cdot \sqrt{\left(1+e^{\frac{\pi\left(-\zeta \omega+\zeta_{1} \omega_{1}-i \omega_{1} \sqrt{1-\zeta_{1}^{2}}\right)}{\omega \sqrt{1-\zeta^{2}}}}\right)^{N}\left(1+e^{\frac{\pi\left(-\zeta \omega+\zeta_{1} \omega_{1}+i \omega_{1} \sqrt{1-\zeta_{1}^{2}}\right)}{\omega \sqrt{1-\zeta^{2}}}}\right)^{N}} \\
= & \lim _{N \rightarrow \infty} O\left(a^{-2 \zeta_{1} N}\right) O\left(b^{-2 N}\right) O\left(c^{N}\right) O\left(d^{N}\right)=0
\end{aligned}
$$

The constants $a, b, c$ and $d$ were used to replace the constants under the square root sign. A more deep analysis of Eq. (16) shows that not only $A_{\text {amp }}$ converges to zero for big values of $N$ for any value of $\omega_{1}$ and $\zeta_{1}$, but also that the convergence is faster for $\zeta_{1}$ higher values.

Even if the consequence of Eq. (16) can appear as the solution to many problems in open-loop control, since the use of a high number of pulses allows a very poor identification of $\omega_{1}$ and $\zeta_{1}$ or their changing due to several causes as observed in the introduction, a drawback has to be considered. The use of $N$ pulses determines an increase in the response time proportional to $N T$, where $T$ is period of the considered mode, and this delay could reduce any advantage in using an open-loop control, one of which positive characteristic is usually the fast response. However, if the method is applied in modal way, the delay effect affects mainly the first mode, since the period $T$ of superior modes is smaller. This suggests a more accurate identification of the frequency and damping in the first mode than in the superior modes in order to limit the number of the pulses.

\section{Conclusions}

In the present paper, on the basis of the availability of general expressions for the time sequence and pulse amplitudes used in motion input pre-shaping, a general sensitivity analysis is performed on the errors than can affect the values of the system parameters and a precise evaluation of the method robustness is given. As follows from the general analytical expression of the response, it is also shown that the use of a high number of pulses allows the modeling errors to be very large and this fact is not very common in open-loop control techniques.

\section{References}

[1] GeorgiaTech, http://www.me.gatech.edu/inputshaping, 2002.

[2] G. Mimmi, L. Frosini, P. Pennacchi and C. Rottenbacher, Open-loop control of a two-links flexible manipulator: experimental results, Proc. of 2001 ASME Design Engineering Technical Conferences and Computer in Engineering Conference, September 9-12, 2001, Pittsburg, PA, on CD-ROM.

[3] K. Ogata, Modern Control Engineering, 3rd ed., Prentice Hall, Englewood Cliffs, NJ, 1997.

[4] L.Y. Pao and W.E. Singhose, On the Equivalence of Minimum Time Input Shaping with Traditional Time-Optimal Control, IEEE Conference on Control Applications, 1995, Albany, NY, pp. 1120-1125.

[5] P. Pennacchi, M. Frontini and G. Mimmi, On Theory of Vibration Control in Linear Systems by Pre-Shaping Motion Input with n-Pulses, Proceedings of the 3rd World Conference on Structural Control, Vol. 2, F. Casciati, ed., John Wiley \& Sons Ltd, Chichester, England, 2002, pp. 613-621.

[6] N.C. Singer and W.P. Seering, Preshaping Command Inputs to Reduce System Vibration, J. of Dynamic Systems, Measurement and Control 112 (1990), 76-82.

[7] O.J.M. Smith, Posicast Control of Damped Oscillatory Systems, Proceedings of the IRE 45 (September 1957), 1249-1255.

[8] C.L. Teo, C.J. Ong and M. Xu, Pulse Input Sequences for Residual Vibration Reduction, Journal of Sound and Vibration 211(2) (1998), $157-177$.

[9] L. Zhe, Increasing Robustness for Minimizing Residual Vibration of Flexible Arms, Proc. of IFToMM Ninth World Congress on the Theory of Machines and Mechanisms, Milan, Italy, August 30-31/September 1-2 1995, pp. 1064-1067. 

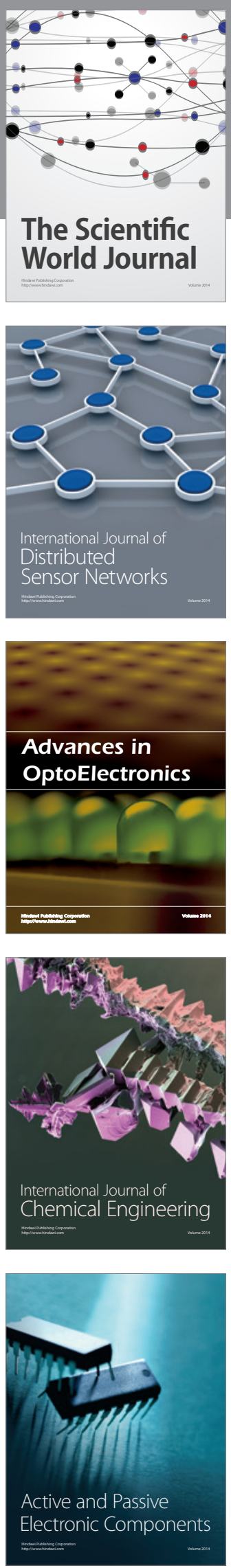
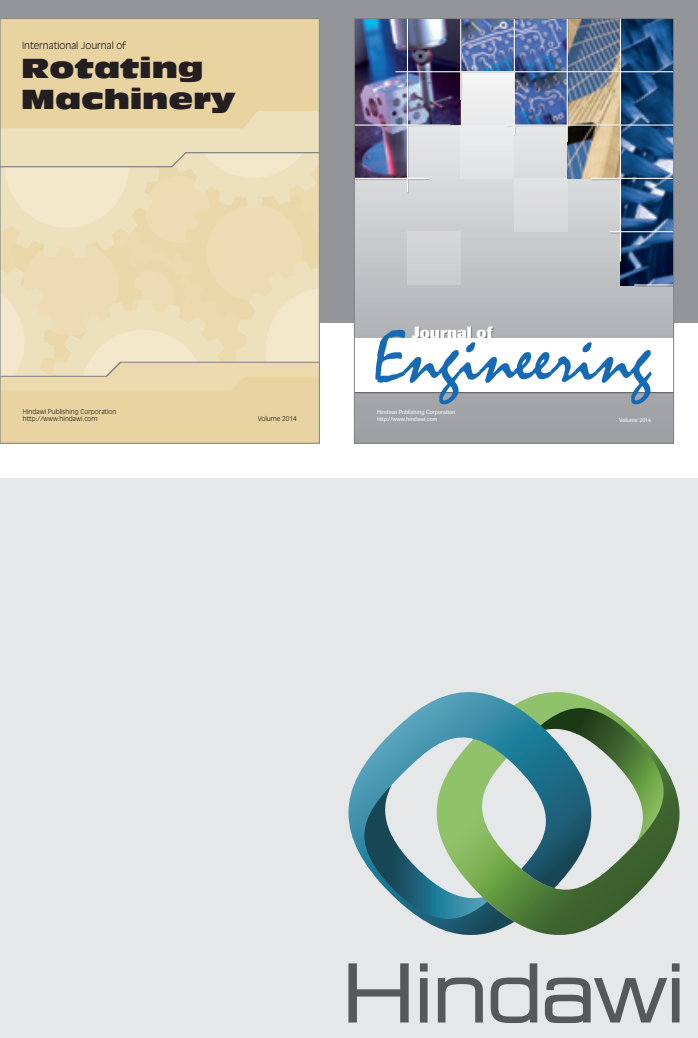

Submit your manuscripts at

http://www.hindawi.com
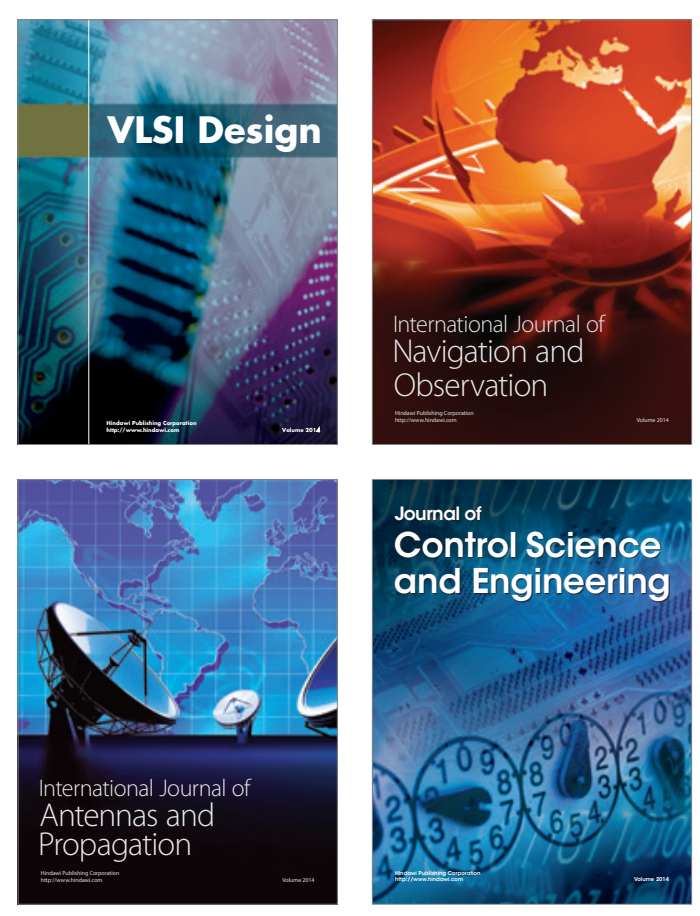
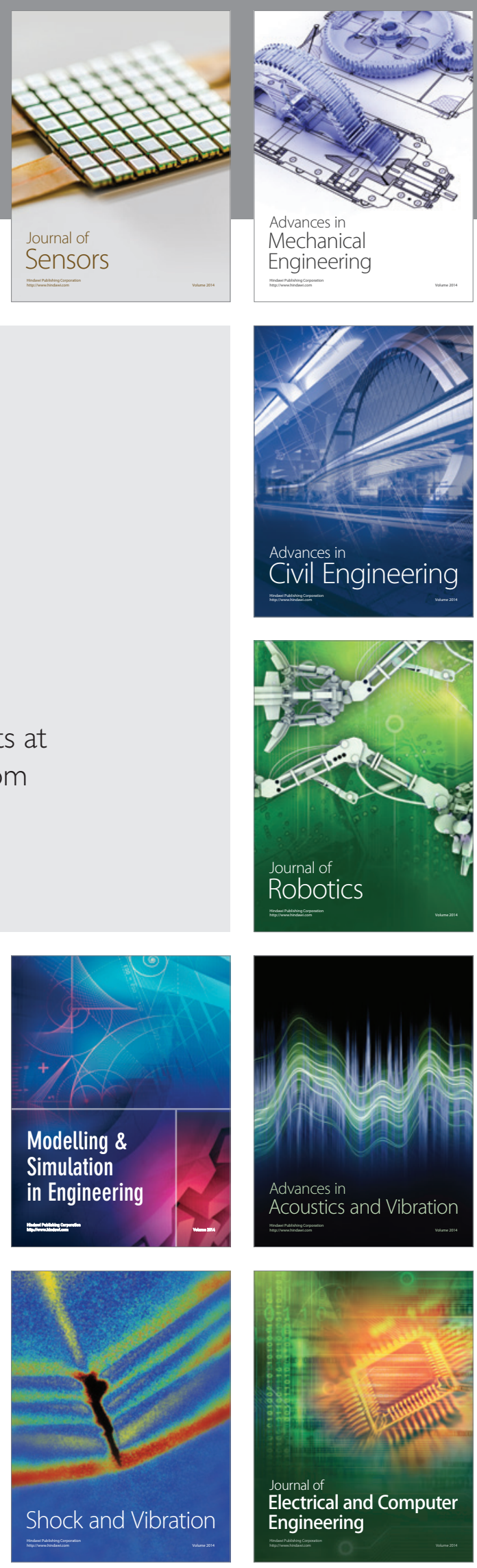